\title{
High-Speed Ultrahigh-Resolution Optical Coherence Tomography Findings in Chronic Solar Retinopathy
}

\author{
Royce W. S. Chen, BA ${ }^{\star}$, Iwona Gorczynska, PHD ${ }^{\star}, \dagger$, Vivek J. Srinivasan, MS ${ }^{\dagger}$, James G. \\ Fujimoto, PHD $†$, Jay S. Duker, MD*, and Elias Reichel, MD* \\ "New England Eye Center, Tufts-New England Medical Center, Tufts University, Boston, \\ Massachusetts \\ tDepartment of Electrical Engineering and Computer Science and Research Laboratory of \\ Electronics, Massachusetts Institute of Technology, Cambridge, Massachusetts
}

\section{Abstract}

Purpose-To describe ocular findings for a 34-year-old man with chronic solar retinopathy using high-speed ultrahigh-resolution (UHR) optical coherence tomography (OCT).

Methods-Fundus photography, fluorescein angiography, and Stratus OCT (Carl Zeiss Meditec, Inc., Dublin, CA) were performed. A high-speed UHR OCT prototype developed in our ophthalmology clinic was used to obtain detailed images of the retina.

Patients-Two eyes of one patient with chronic solar retinopathy were studied.

Results-Both Stratus OCT and high-speed UHR OCT demonstrated foveal thinning bilaterally. In addition, high-speed UHR OCT showed distinct hyporeflective disruptions in the photoreceptor inner segment/outer segment junction and photoreceptor outer segments bilaterally. En face OCT images from three-dimensional OCT data sets revealed hyporeflective regions of photoreceptor atrophy in the outer retina.

Conclusions-High-speed UHR OCT showed more detail than standard OCT, and findings were consistent with histopathologic and ultrastructural features that have been reported previously. Solar retinopathy should be studied further with high-speed UHR OCT to determine the short- and longterm effects of solar radiation damage.

\section{Keywords}

optical coherence tomography; solar retinopathy; ultrahigh-resolution optical coherence tomography

Solar retinopathy is a condition characterized by central visual distortion in patients who have a history of sun gazing. Although most cases are commonly attributed to solar eclipse viewing, damage from sun gazing has also been described in religious ritual participants, military personnel, people with mental disturbances, and sunbathers. ${ }^{1}$ We examined a patient with solar retinopathy using high-speed ultrahigh-resolution (UHR) optical coherence tomography (OCT).

Reprint requests: Elias Reichel, MD, Ophthalmology Department, Tufts-New England Medical Center, 750 Washington Street, Boston, MA 02111; EReichel@tufts-nemc.org.

E. R. had full access to all the data in the study and takes responsibility for the integrity of the data and the accuracy of the data analysis. J. G. F. receives royalties from intellectual property licensed by MIT to Carl Zeiss Meditec, Inc. J.S.D. receives research support from Optovue, Inc. 


\section{Case Report}

A 34-year-old man from Haiti was referred for complaints of blurred vision of 15 years' duration in both eyes. In the past, as part of a religious ritual, he stared at the sun with both eyes for several minutes at a time on multiple occasions; however, he had not practiced this for the past several years. Best-corrected visual acuity was $20 / 40$ in both eyes. Dilated fundus examination revealed a well demarcated circular lesion in his foveae (Fig. 1A, yellow arrow). Fluorescein angiography findings were normal bilaterally. OCT (Stratus OCT; Carl Zeiss Meditec, Inc., Dublin, CA) demonstrated central foveal thinning bilaterally (central 1-mm-diameter ring thickness: right eye, $143 \mu \mathrm{m}$; left eye, $131 \mu \mathrm{m}$ ). There was evidence of early posterior hyaloid separation in both eyes, but the vitreoretinal interface was otherwise normal bilaterally.

We performed high-definition and three-dimensional retinal imaging using a high-speed UHR OCT clinical prototype capable of $3.5-\mu \mathrm{m}$ axial resolution. ${ }^{2}$ Although the inner retina appeared normal, the outer nuclear layer was almost entirely absent in the fovea, contributing to the thin foveal measurements quantified on Stratus OCT. In the fovea, there were distinct hyporeflective disruptions in the photoreceptor inner segment/outer segment junction and photoreceptor outer segments, which were more pronounced in the right eye than in the left eye (Fig. 1D, white brackets). Notably, the external limiting membrane, retinal pigment epithelium (RPE), and Bruch membrane/choriocapillaris complex appeared normal (Fig. 1, C and D). Dense raster scan three-dimensional OCT data were acquired and used to generate en face OCT fundus images. By sectioning and displaying only the reflections from the outer retina between the external limiting membrane and the RPE (Fig. 2), photoreceptor features could be selectively viewed. Hyporeflective areas (outlined in yellow) indicated areas of photoreceptor disruption, where the total reflectivity of the outer retina was reduced. The dark, hyporeflective area was slightly larger and more continuous in the right eye than in the left eye (Fig. 2).

\section{Discussion}

Three histopathologic reports in the literature demonstrated that ocular injury resulting from solar retinopathy is predominantly limited to the outer retina. ${ }^{1,3,4}$ Our observations using highspeed UHR OCT correlated well with these histopathologic reports. Although the inner retina appeared normal, the outer nuclear layer in the foveal region was almost entirely absent bilaterally, and vertical bands of hyporeflectivity through the photoreceptor inner segment/ outer segment junction and outer segments demonstrated focal areas of photoreceptor cell death.

One histopathologic report by Tso and La Piana ${ }^{1}$ described findings of widespread RPE changes after sun gazing, while another report by Hope-Ross et $\mathrm{al}^{3}$ found the outer segments of the photoreceptors to be most susceptible to solar radiation damage with relatively less RPE involvement. Both of these studies examined eyes that had been enucleated within 1 week of solar gazing. This was in contrast to our patient, who was several years removed from his last exposure. In the high-speed UHR OCT image, the hyperreflective line representing the RPE did not appear to be affected: this finding suggests either that the RPE was not significantly damaged or that the RPE had recovered after the acute injury phase. In agreement with the report by Hope-Ross et al, ${ }^{3}$ the most prominent changes in our patient were found in the photoreceptor outer segments.

Two previous standard-resolution OCT reports described areas of hyporeflectivity in the outer retina of patients with chronic solar retinopathy. ${ }^{5,6}$ Although our results were similar to these reports, high-speed UHR OCT revealed some areas of normal reflectivity between and immediately adjacent to the hyporeflective bands of atrophy. This finding likely demonstrates 
varying degrees of photoreceptor survival as a result of minor movements of the sun's image on the retina during sun gazing. The report by Garg et al ${ }^{5}$ suggested involvement of the RPE based on OCT images, but on closer inspection of the standard OCT images as well as our high-speed UHR OCT images, it appears that the RPE was spared.

It is interesting that the hyporeflective areas in the en face images were similar in shape for both eyes, with slightly greater involvement in the right eye than in the left eye. This finding suggests two points. First, because the patient gazed at the sun with both eyes open, he had a similar distribution of photoreceptor atrophy in both eyes, and second, as mentioned in a previous report on acute solar retinopathy, the right (and dominant) eye had a greater area of involvement than the left eye. ${ }^{7}$

In conclusion, our results agree with histopathologic and previous OCT reports that chronic damage from sun gazing is primarily confined to the outer retina. The increased axial resolution of our prototype instrument demonstrated focal bands of atrophy that could not be visualized by standard-resolution OCT. More patients with different stages of solar retinopathy should be studied with high-speed UHR OCT to determine both short- and long-term effects of solar radiation damage.

\section{Acknowledgments}

Supported in part by National Institutes of Health (RO1-EY11289-20), Air Force Office of Scientific Research, Medical Free Electron Laser Program (contract FA9550-040-1-0046, FA9550-040-1-0011), National Science Foundation (BES-0522845), an unrestricted grant from Research to Prevent Blindness, Inc., and a Medical Student Eye Research Fellowship.

\section{References}

1. Tso MO, La Piana FG. The human fovea after sungazing. Trans Am Acad Ophthalmol Otolaryngol 1975;79:788-795.

2. Wojtkowski M, Srinivasan VJ, Fujimoto JG, et al. Three-dimensional retinal imaging with high-speed, ultrahigh-resolution optical coherence tomography. Ophthalmology 2005;112:1734-1746. [PubMed: 16140383]

3. Hope-Ross MW, Mahon GJ, Gardiner TA, et al. Ultrastructural findings in solar retinopathy. Eye 1993;7:29-33. [PubMed: 8325420]

4. Gass, JDM. Stereoscopic Atlas of Macular Diseases: Diagnosis and Treatment. Vol. 4th. St. Louis: Mosby; 1997. p. 760-763.

5. Garg SJ, Martidis A, Nelson ML, Sivalingam A. Optical coherence tomography of chronic solar retinopathy. Am J Ophthalmol 2004;137:351-354. [PubMed: 14962431]

6. Jorge R, Costa RA, Quirino LS, et al. Optical coherence tomography findings in patients with late solar retinopathy. Am J Ophthalmol 2004;137:1139-1143. [PubMed: 15183809]

7. Yannuzzi LA, Fisher YL, Krueger A, Slakter J. Solar retinopathy: a photobiological and geophysical analysis. Trans Am Ophthalmol Soc 1987;85:120-158. [PubMed: 3328915] 

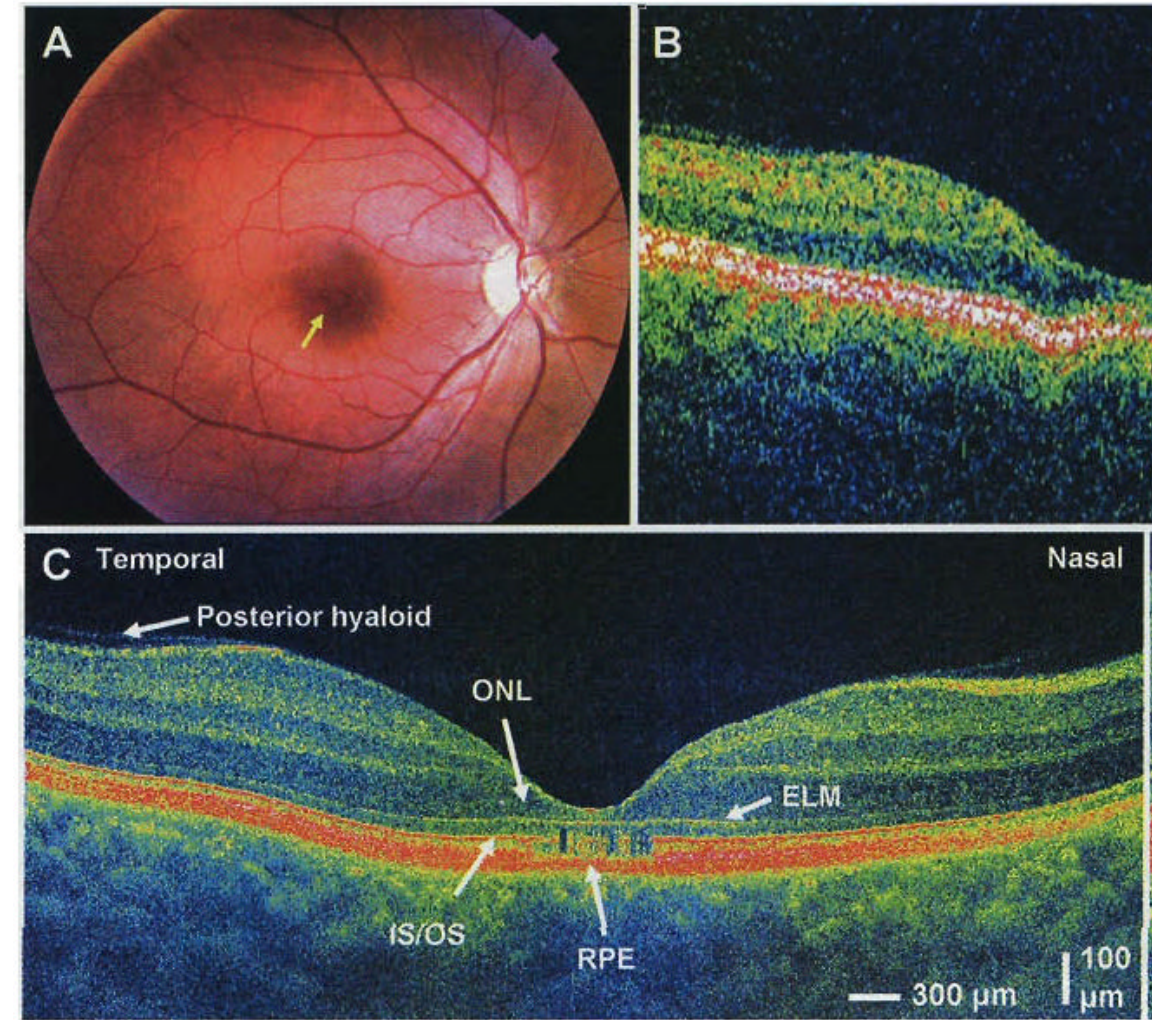
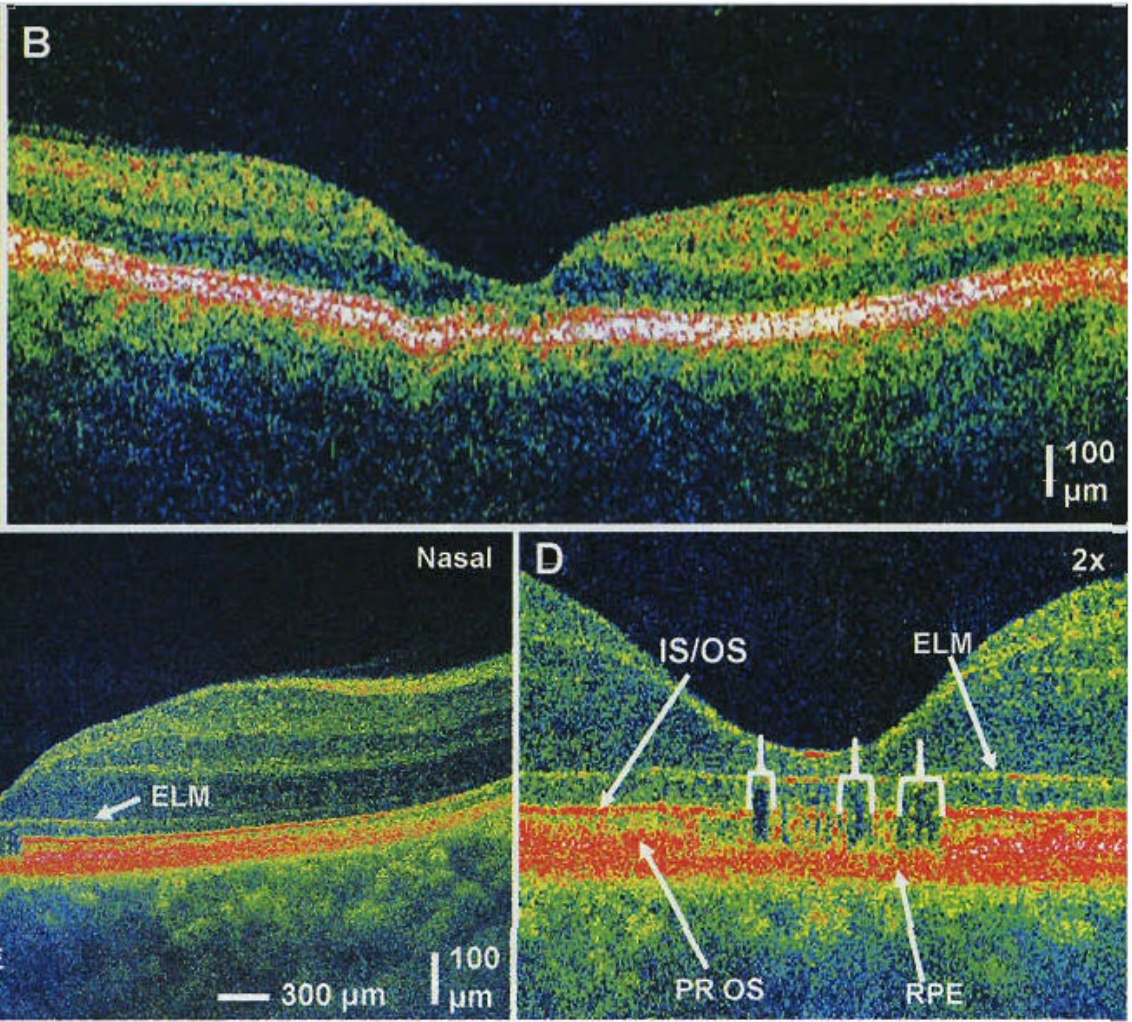

Fig. 1.

A, Color photograph of the right eye. A well demarcated circular lesion is present in the fovea (yellow arrow). B, Horizontal standard 10- $\mu$ m-resolution (Stratus OCT [optical coherence tomography]) OCT image of the right eye. The foveal thickness (central 1-mm-diameter ring) is $143 \mu \mathrm{m}$. C, Horizontal high-speed ultrahigh-resolution (UHR) OCT image of the right eye with 3.5- $\mu \mathrm{m}$ axial resolution and 8,192 axial scans. Early posterior hyaloid separation is visible (white arrow). The outer nuclear layer is significantly thinned and is almost entirely absent centrally. ONL, outer nuclear layer; IS/OS, photoreceptor inner segment/outer segment junction; ELM, external limiting membrane; RPE, retinal pigment epithelium. D, Two times magnification of the high-speed UHR OCT image. Vertical hyporeflective bands disrupting the IS/OS junction and photoreceptor outer segments (PR OS) are clearly visible, interspersed with areas of normal reflectivity. 

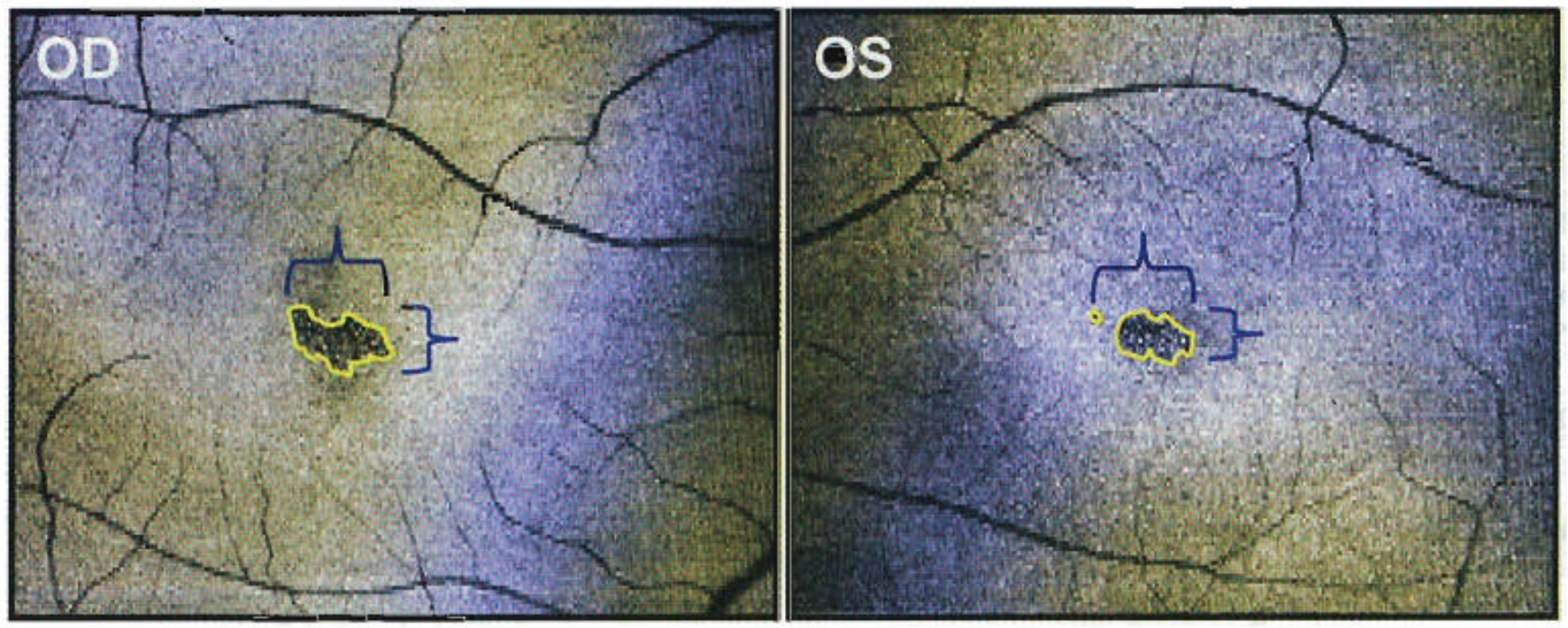

Fig. 2.

En face images of the reflectivity of the outer retina show a similar pattern of hyporeflectivity in both eyes where the photoreceptors are disrupted (yellow outline). The area of involvement is greater in the right eye. 